\title{
A leather coating with self-healing characteristics
}

\author{
Feifei Liang, Tianyou Wang, Haojun Fan*, Jun Xiang and Yi Chen
}

\begin{abstract}
Genuine leather is often coated before making daily necessities such as shoes, clothing, bags, sofas, car seats, etc., so as to impart leather products various colours, higher wear resistance and water resistance and so on. However, the coating of these products is often damaged in daily use which will decrease its aesthetic effect and practicability. Therefore, how to improve the scratch resistance of leather coatings has been puzzling people all the time. It is a common knowledge that animals and plants can repair the injured biological tissues by himself. According to this principle, here, we prepared a type of self-healing water-borne polyurethane with disulfide bond in the main chain by using HEDS as chain extender, and the self-healing system was triggered by the disulfide bonds with the help of shape memory function of waterborne Polyurethane, self-healing experiments how that the damaged of leather coating can be repaired fully at $60^{\circ} \mathrm{C}$ for $12 \mathrm{~h}$.
\end{abstract}

Keywords: Waterborne polyurethane, Self-healing, Leather coating

\section{Introduction}

Genuine leather is widely favored by people because of its gorgeous and fashionable appearance, soft and exquisite hand feeling, excellent physical and mechanical properties as well as outstanding wearing comfort. However, leather products inevitably suffer from physical damages, the occurred scratches will destruct the appearance and reduce the lifespan of products.

According to the self-healing process of damaged biological tissue, injured tissue and cells are repaired by regeneration of adjacent healthy cells to restore tissue integrity. Inspired by bionics, up to know, there are two type of self-healing materials named as extrinsic and intrinsic self-healing materials [1]. The extrinsic self-healing material depends on the healing agents embedded in the material, which are often stored in microcapsules, particles, or a microvascular network. When the material is damaged, the healing agent can flow out to quickly restore the material to its original state in a short time, whereas this system enables only one-time healing at a certain place [2]. Intrinsic self-healing materials based on reversible bonds can effectively solve this problem, these chemical bonds include noncovalent and dynamic covalent bonds, which can be stimulated by moderate external energy (e.g. light, heat). In contrast to external self-repairing materials, internal self-repairing materials can achieve multiple repairs of the same damaged site. Therefore, intrinsic self-repairing materials be given a growing number of attention in recent years [3-5] (Table 1).

Nowadays, most of the intrinsic self-healing materials are based on dynamic chemical reversible bonds such as disulfide bond, Diels-Alder (DA) bonding, acylhydrazone bond and hydrogen bonding, etc. Among all these reversible chemical bonds, disulfide bond is the best candidate for self-healing materials because it can undergo chain exchange reaction under heat, UV light, and redox conditions [6, 7]. Distinguished from most of other available reversible reactions, moderate temperature (about $60^{\circ} \mathrm{C}$ ) can initiate

* Correspondence: fanhaojun@scu.edu.cn

Key Laboratory of Leather Chemistry and Engineering of Ministry of

Education, Sichuan University, Chengdu 610065, People's Republic of China 
Table 1 mechanical properties variation of polyurethane film before and self-healing

\begin{tabular}{lll}
\hline Parameter & Tensile strength/MPa & Elongation at break/\% \\
\hline Original sample & 11.49 & 754 \\
Self-healing sample & 9.25 & 605 \\
Self-healing efficiency & $80.5 \%$ & $80.2 \%$
\end{tabular}

chain exchange reaction of disulfides, which provides a convenient condition for self-healing of materials. Many researchers have designed a variety of selfhealing polymers to meet the requirements of different applications based on the characteristics of disulfide bonds.

The whole self-healing mechanism of polymers containing disulfide bonds was presented in Fig. 1. When the crack take placed, the shape memory effect of the polymer causes the fracture to link together. At the suitable temperature, the system achieves the bond dissociation energy of disulfide bonds, these bonds dissociate to take shape radicals and attack adjacent bonds, leading to the formation of new S-S bonds. Therefore, Polymers containing disulfide bond can almost restore their original mechanical properties and heal the surface damage of coatings at suitable temperature for a period of time though the collaboration of disulfide exchange reaction and shape memory effect [8].

Through continuous exploration, people have not only synthesized self-healing inorganic materials such as concrete, ceramics and metals [9], but also developed different types of organic polymeric self-healing materials, such as polyurethane, hydrogel and so on. However, there is few report on the leather with selfhealing property. As we know, leather is made up of coating layer and genuine leather base, the self- healing property of leather should be dependent on coating materials. As a typical coating materials, waterborne polyurethane (WPU) have found extensive commercial applications in various substrates such as genuine leather, artificial leather, textile, plastics, wood as well as ceramics by virtue of their environment-friendly characteristics, outstanding performances and remarkable versatility. On this base, here a type of self-healing water-borne polyurethane with disulfide bond in the main chain was prepared firstly, then the self-healing characteristics of coating layer of leather was investigated.

\section{Main text}

I The synthetic process of self-healing waterborne polyurethane was as follows. First, bismuth acid catalyst, isophorone diisocyanates (IPDI), polyoxytetramethylene glycol (PTMG) and trimethylolpropane (TMP) were added to a flask fitted with a mechanical stirrer and a thermometer. The reaction was carried out under a nitrogen atmosphere at $75 \sim 85^{\circ} \mathrm{C}$ for $1 \mathrm{~h}$, and then 2-hydroxyethyl disulfide (HEDS) was added for another $1 \mathrm{~h}$ at $55^{\circ} \mathrm{C}$. Subsequently, dimethylol propionic acid (DMPA) neutralized by three ethylamine (TEA) is fed into the reactor, and allowed to react $3 \mathrm{~h}$ until the residual $-\mathrm{NCO}$ groups reach the theoretical amount. Finally, a large amount of deionized water is fed into the vessel to obtain a certain solid content emulsion. The post chain extension is performed with 3-(2-aminoethylamino) propyl dimethoxy methyl silane (KH602) under vigorous stirring. The synthesis route is shown in Fig. 2.

According to this method, disulfide bond was successfully introduced into the main chain of waterborne polyurethane. Compared with the common polyurethane, the disulfide bond endows the polyurethane with good self-healing properties. Mechanical properties can be

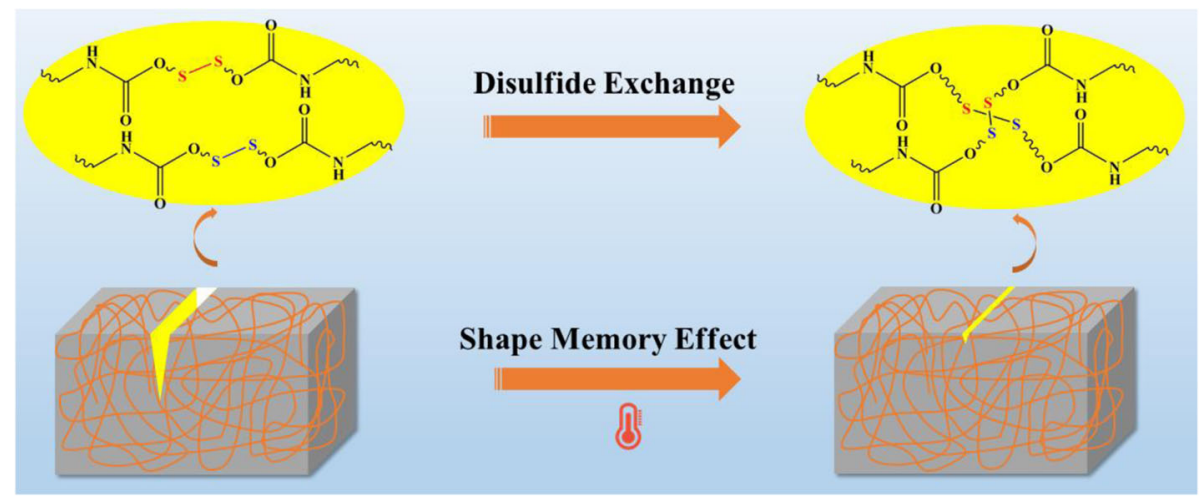

Fig. 1 Illustration of the healing process of the self-healing polyurethane 


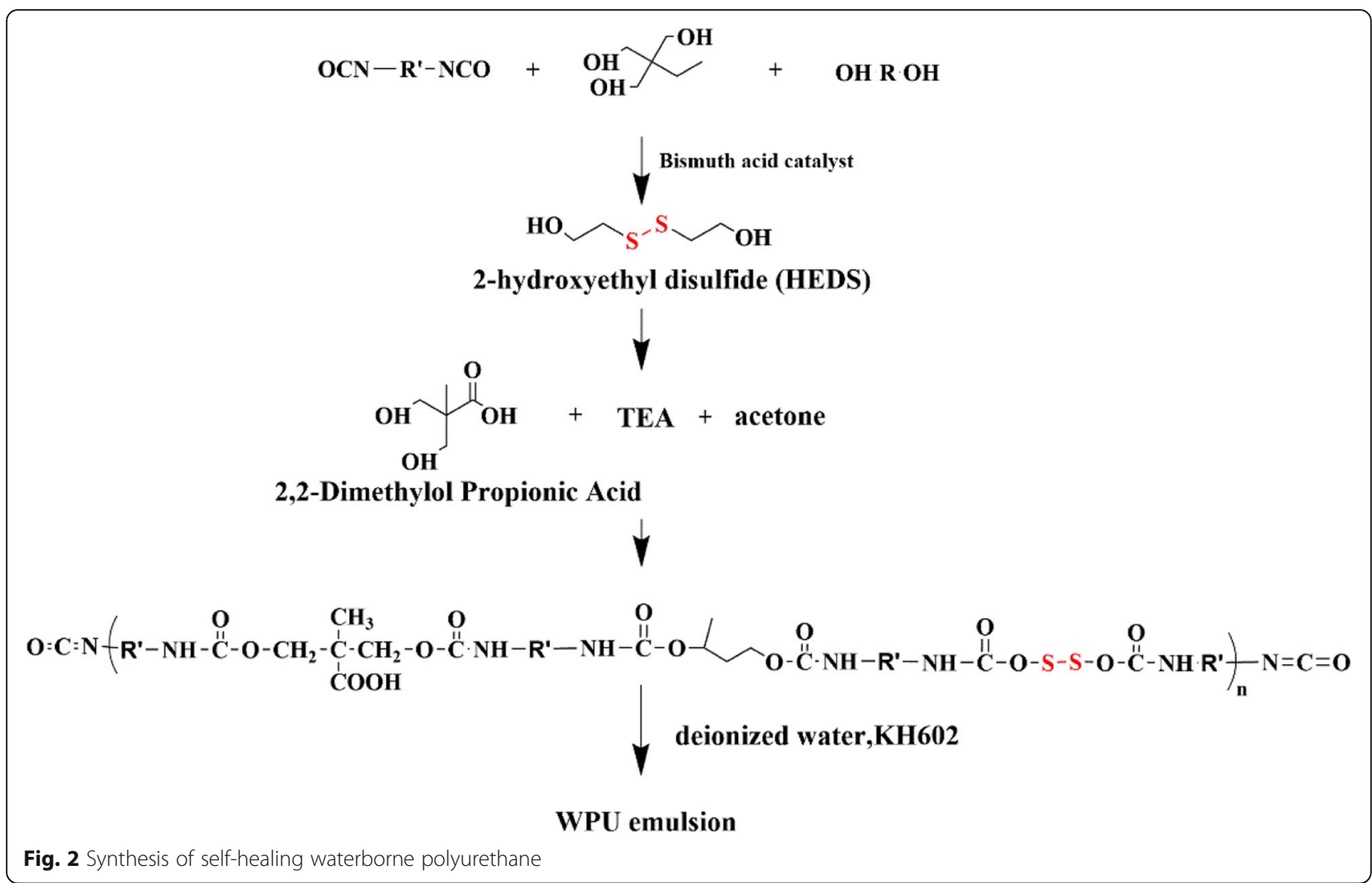

used to characterize the self-healing properties of waterborne polyurethane film. In experiment, the polyurethane film is cut into dumbbell-shaped according to the specifications and divided into two parts, one part is used as the original sample to test the tensile strength. Another part is cut with medical scissors from the middle of the neck of the specimen firstly, then assembled immediately and placed in the container. Finally, according to the experimental conditions, the specimens are placed in an oven at $60^{\circ} \mathrm{C}$ for $12 \mathrm{~h}$, then taken out for testing the mechanical properties variation, and the maximum tensile strength and elongation at break are recorded. Comparing the mechanical properties of healed spline with that of original spline, the self-healing efficiency is obtained.

As shown in Fig.3. The original sample exhibited a tensile strength of $11.49 \mathrm{MPa}$ and elongation at break of $754 \%$, after $12 \mathrm{~h}$ at $60^{\circ} \mathrm{C}$, the tensile strength and elongation at break of healed sample change to 9.25 $\mathrm{MPa}$ and $605 \%$ respectively. The self-healing ratios are $80.5 \%$ and $80.2 \%$ respectively. This indicates that the disulfide exchange reaction reconstructs the chemical bonds on the crack surface during healing process, thus effectively restoring the mechanical properties of polyurethane films $[10,11]$. Shape memory effect plays an assistant restoring role in the process of self-healing, it can help the scratch surface of macroscale approach and make the exchange reaction of disulfide start quickly, but it has little effect on the recovery of mechanical properties.

Similarly, the coating layer of leather was scratched with medical blade, and then put into the oven at $60^{\circ} \mathrm{C}$ for $12 \mathrm{~h}$, the results are shown in Fig.4. It can be seen that the scratch have almost disappeared, showing a remarkable self-healing characteristics In process of heating, the waterborne polyurethane could recovery its original shape and brought the crack surfaces connective. Disulfide bonds can be broken down by dissociation of lower bonds, and the splitting free radicals are rearranged with adjacent molecular chains to form new disulfides to heal scratches [11].

In order to observe the self-healing process of leather coating directly, scanning electron microscopy (SEM) was used to magnify the changes of scratch. The results show that the cracks of the original leather coating were wider, but the gap becomes smaller and smaller as time goes on. After self-healing for $12 \mathrm{~h}$, the gap of scratches is fully healed. According to the self-healing efficiency and the healing effect of leather coating, the as-prepared self-healing polyurethanes not only 


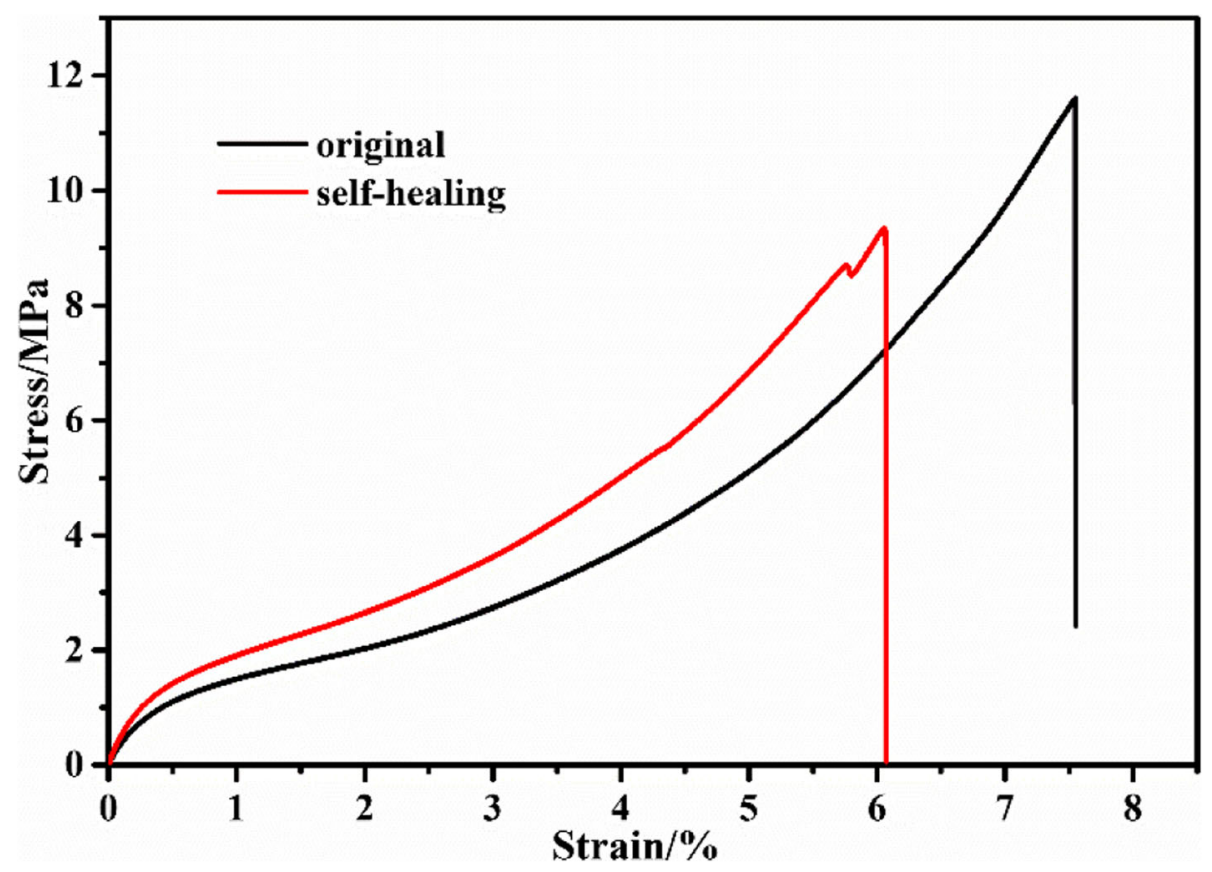

Fig. 3 Comparison of mechanical properties of polyurethane original and self-healing

exhibited excellent mechanical properties, but also could quickly restore the original performances without external intervention at a moderate temperature after damaging.

\section{Conclusions}

In conclusion, a water-borne polyurethane with disulfide bond in the main chain was prepared by using HEDS as chain extender, which exhibited excellent mechanical property and self-healing ability. Up heating, the shape memory effect combines the fracture surfaces together, the disulfide exchange reaction in the system is the main reason for the self-healing process. Through mechanical propertiestests, the waterborne polyurethane films exhibit higher self-healing properties, its self-healing efficiency can reach to $80 \%$. When used as coating materials, the finished leathers also show excellent selfhealing characteristic, the scratches on the surface can fully healed at $60^{\circ} \mathrm{C}$ for $12 \mathrm{~h}$. This smart materials can almost fully heal the damaged coating and shows broad application in coating, leather/synthetic leather finishing, textile laminating and so on.

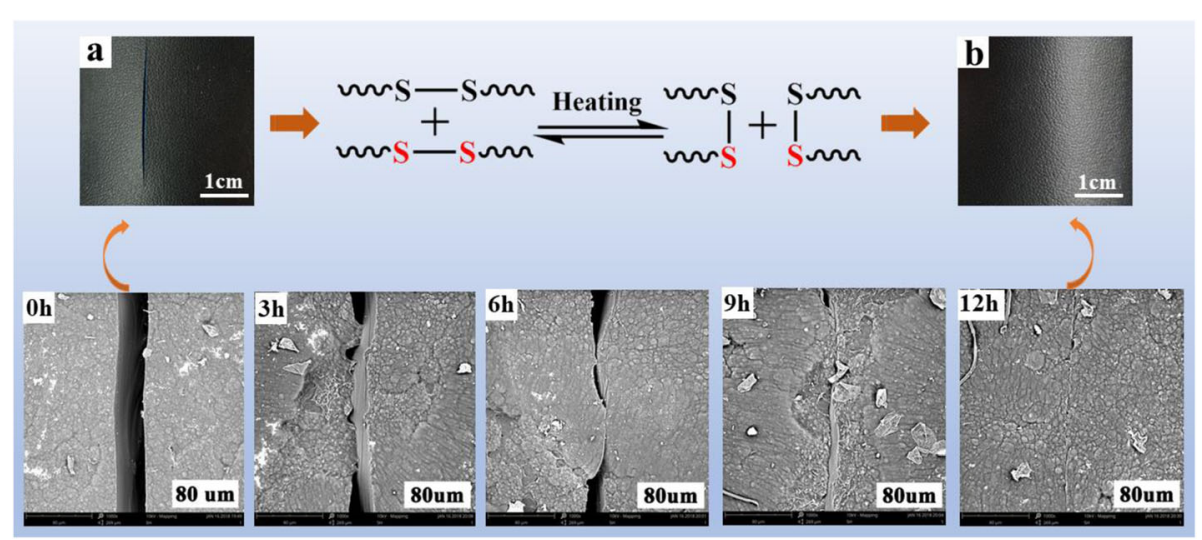

Fig. 4 Self-healing process of leather coating: (a) damaged leather coating; (b) leather coating after be repaired at $60^{\circ} \mathrm{C}$ for $12 \mathrm{~h}$; SEM image is the self-healing process of damaged leather coating 


\section{Supplementary information}

Supplementary information accompanies this paper at https://doi.org/10. 1186/s42825-020-0018-4

Additional file 1. Supporting Information. Figure S1. NMR Carbon Spectrum Analysis of 2-hydroxyethyl disulfide (HEDS). Figure S2. Particle size analysis of self-healing waterborne polyurethane emulsion.

\section{Acknowledgements}

We appreciate Wang Zhonghui from College of Light Industry, Textile \& Food Engineering of Sichuan University for her great help in SEM observation.

\section{Authors' contributions}

FL synthesized the self-healing polyurethane, interpreted the data, and was a major contributor in writing the manuscript. TW performed the emulsion particle size. YC performed the mechanical strength of polyurethane films. JX calculated the self-repairing efficiency of polyurethane. HF performed the SEM experiments. All authors read and approved the final manuscript.

\section{Funding}

This work was supported by the National Key Research and Development Program of China (Project No: 2017YFB0308600).

\section{Availability of data and materials}

All data generated or analysed during this study are included in this published article [and its supplementary information files].

\section{Competing interests}

The authors declare that they have no competing interests.

Received: 19 June 2019 Accepted: 23 January 2020

Published online: 29 February 2020

\section{References}

1. Wan T, Chen D. Synthesis and properties of self-healing waterborne polyurethanes containing disulfide bonds in the main chain. J Mater Sci. 2016:52(1):197-207.

2. Toohey KS, Sottos NR, Lewis JA, et al. Self-healing materials with microvascular networks. Nat Mater. 2007;6(8):581-5.

3. Lai Y, Kuang $X$, Zhu P, et al. Colorless, transparent, robust, and fast scratchself-healing elastomers via a phase-locked dynamic bonds Design. Adv Mater. 2018;30(38):1802556.

4. Xiao $Y$, Huang $H$, Peng $X$. Synthesis of self-healing waterborne polyurethanes containing sulphonate groups. RSC Adv. 2017;7(33):20093-100.

5. Yang GW, Zhang YY, Wang Y, et al. Construction of autonomic self-healing $\mathrm{CO}_{2}$-based polycarbonates via one-pot tandem synthetic strategy. Macromol. 2018. https://doi.org/10.1021/acs.macromol.7b02715.

6. Jian X, Hu Y, Zhou W, et al. Self-healing polyurethane based on disulfide bond and hydrogen bond. Polym Advan Technol. 2017. https://doi.org/10 1002/pat.4135.

7. Xu Y, Chen D. A novel self-healing polyurethane based on disulfide bonds. Macromol Chem Phys. 2016;217(10):1191-6.

8. Ling L, Li J, Zhang G, et al. Self-healing and shape memory linear polyurethane based on disulfide linkages with excellent mechanical property. Macromol Res. 2018;26(4):365-73.

9. Feula A, Tang X, Giannakopoulos I, et al. An adhesive elastomeric supramolecular polyurethane healable at body temperature. Chem Sci. 2016;7(7):4291-300.

10. Yang Y, Lu X, Wang W. A tough polyurethane elastomer with self-healing ability. Mater Des. 2017;127:30-6.

11. Deng $X Y$, Xie $H$, Du L, et al. Polyurethane networks based on disulfide bonds: from tunable multi-shape memory effects to simultaneous selfhealing. Sci China Mater. 2019;62(3):437-47.

\section{Publisher's Note}

Springer Nature remains neutral with regard to jurisdictional claims in published maps and institutional affiliations.

\section{Submit your manuscript to a SpringerOpen ${ }^{\circ}$ journal and benefit from:}

- Convenient online submission

- Rigorous peer review

- Open access: articles freely available online

- High visibility within the field

- Retaining the copyright to your article

Submit your next manuscript at $\boldsymbol{\nabla}$ springeropen.com 